\title{
Obesidad y cáncer: fisiopatología y evidencia epidemiológica
}

\section{Jefersson David Sepúlveda Fonseca ${ }^{1}$, Md. Ricardo Andrés Quintero Farías ${ }^{2}$}

1 Estudiante de sexto año de medicina humana. Escuela de Medicina. Facultad de Ciencias de la Salud. Universidad Pedagógica y Tecnológica de ColombiaUPTC. Tunja. Boyacá. Colombia.

2 Médico cirujano. Especialista en Medicina Interna. Docente de Medicina Interna. Universidad Pedagógica y Tecnológica de Colombia - UPTC. Tunja. Boyacá. Colombia.

Correo electrónico: jdse10@hotmail.com

Fecha de recepción: Noviembre 26 de 2015

Fecha de corrección: Febrero 23 de 2016

Fecha de aprobación: Marzo 16 de 2016

\section{Resumen}

Introducción: La obesidad es reconocida como un importante problema de salud pública en todo el mundo, la prevalencia de sobrepeso y obesidad está aumentando a un ritmo alarmante tanto en países desarrollados como en vía de desarrollo, tanto así, que la obesidad ha sido considerada como la epidemia del siglo XXI. El exceso de peso corporal es un factor de riesgo importante en la aparición de enfermedades crónicas y determinados tipos de cáncer, tales como el cáncer de colon, mama en mujeres menopáusicas, endometrio, riñón y cáncer de esófago. Métodos: En junio del 2015, se realizó una búsqueda sistemática de la literatura científica en las principales bases de datos biomédicas: PUBMED, EMBASE, MEDLINE, LILACS, IMBIOMED, COCHRANE, entre el año 1980 y 2015. Utilizando las siguientes palabras clave: cáncer, obesidad, neoplasia, sobrepeso, epidemiologia, asociación y revisión. Resultados: se encontró que existe una asociación estadísticamente significativa entre la obesidad y diversos tipos de cáncer, tales como el cáncer de colon, de mama en mujeres posmenopáusico, de endometrio, de riñón, y de esófago. La obesidad y el sobrepeso pueden tener efectos notables sobre varios sistemas fisiológicos que pueden posteriormente aumentar el riesgo de cáncer. Conclusión: Existe una asociación entre la obesidad y la aparición de diversos tipos de cáncer, la comprensión de los mecanismos causales de esta asociación es importante para la implementación de medidas de salud pública y cambios en el estilo de vida, que busquen prevenir la aparición del cáncer y la obesidad. Palabras clave: cáncer, obesidad, neoplasia, sobrepeso, epidemiologia, asociación y revisión.
Obesity and cancer: pathophysiology and epidemiological evidence

Background: Obesity is said to be a major public health issue around the globe. The prevalence of overweight and obesity is increasing at an alarming rate in developed and developing countries, to the extend that obesity is being regarded as the epidemic of the XXI century. Overweight is an important risk factor in the acquisition of chronic diseases and certain types of cancer such as; colorectal cancer, esophageal cancer, endometrial cancer, kidney cancer, and breast cancer in menopausal women. Methods: In June 2015, a systematic search for scientific literature in the main biomedical databases was performed: PUBMED, EMBASE, MEDLINE, LILACS, IMBIOMED and COCHRANE, making use of the following key words: cancer, obesity, neoplasm, overweight, epidemiology, associations and review, between 1980 and 2015. Results: There is a significant statistical relationship between obesity and several types of cancer, such as; colorectal cancer, esophageal cancer, endometrial cancer, kidney cancer, and breast cancer in post-menopausal women.Obesity and overweight may have considerable effects on several physiological systems that may subsequently increase the risk of cancer. Conclusions: There is a relationship between obesity and the onset of several types of cancer. The understanding of this mechanism is important for the implementation of public health measures and changes in life style, seeking to prevent cancer and obesity.

Keywords: cancer, obesity, neoplasm, overweight, epidemiology, associations y review.

\section{Introducción}

La obesidad es reconocida como un importante problema de salud pública en todo el mundo (1). La prevalencia de sobrepeso y obesidad está aumentando a un ritmo alarmante tanto en países desarrollados como en vía de desarrollo (2), tanto así, que la obesidad ha sido considerada por la comunidad médica como la epidemia del siglo XXI (3).

Desde de la década de los 80, la obesidad se ha duplicado en el mundo. Para el año 2015 la organización mundial de la salud (OMS), estima que 2300 millones de adultos tendrán sobrepeso y más de 700 millones de personas serán obesas. La epidemia de la obesidad no solo se limita a la población adulta, estudios recientes han determinado que la población pediátrica también se está viendo notablemente afectada. En el año 2010 , alrededor de 40 millones de niños menores de cinco años tenían sobrepeso. En Colombia, se observa un importante aumento en la prevalencia de sobrepeso y obesidad en adultos. La prevalencia de sobrepeso tiene un promedio de $32 \%$ en personas de 18 a 64 años de edad, sin diferencia entre géneros. Sin embargo, la prevalencia de obesidad es casi el doble en mujeres (16.6 \%) que en hombres (8.8 \%). La Encuesta Nacional sobre la Situación Nutricional y de Consumo de Alimentos en Colombia (ENSIN) 2010 reporta que el 13.4\% de la población entre 5 y 17 años presenta sobrepeso y el $4.1 \%$ obesidad; el sobrepeso predomina en las niñas y en los niños la obesidad (4-7).

El exceso de peso corporales un factor de riesgo importante en la aparición de enfermedades cardiovasculares, trastornos musculo-esqueléticos, dislipidemias, diabetes mellitus tipo 2, trastornos en las vías respiratorias, enfermedades articulares (8) y determinados tipos de cáncer (9).

El cáncer es una de las principales causas de muerte en el mundo. El aumento previsto de cáncer a nivel mundial pasara de 12.7 millones de nuevos casos en el año 2008 a 22.2 millones en el año 2030 (10-16). En Colombia, el doctor Piñeros y colaboradores, analizaron la tasa de 
mortalidad de cáncer en el país tanto para el género femenino como para el masculino, en un periodo comprendido entre los años 1984 y 2008, y determinaron que en el género sexo masculino las cinco primeras causas de muerte de origen neoplásico fueron el cáncer de estómago (17.6\%), próstata (15.0\%), pulmón (14.8\%), colorrectal $(6.5 \%)$ y la leucemia (5.3\%). En el sexo femenino, las causas más comunes de muerte de origen neoplásico fueron el cáncer de mama (12.3\%), cuello uterino (12.1\%), estómago (11.5\%), pulmón (9.2\%) y cáncer colorrectal (7.6\%) (17-20).

El objetivo de este artículo fue determinar la asociación epidemiológica y fisiopatológica que existe entre la obesidad y los diferentes tipos de cáncer documentados en la literatura médica.

\section{Materiales y métodos:}

En junio del 2015 se adelantó una revisión sistemática de todos los artículos disponibles en PubMed, Medline, Cochrane, Lilacs, Imbiomed y Embase. Se limitó la búsqueda a trabajos originales, revisiones de tema y metaanálisis, publicados en inglés y español, sin límite en edad o sexo, desde 1980 a junio de 2015, utilizando la combinación de los siguientes términos según el MESH (medical subject headings): cancer, obesity, neoplasms, Overweigh, Epidemiology, associationsy review.

Se hizo la lectura inicial del resumen de 280 trabajos de los cuales se seleccionaron 69 que eran representativos para el tema de esta revisión. Se procedió luego a la consecución de los artículos completos, su lectura, complementación con artículos relevantes, y por último al ordenamiento, clasificación y redacción del texto.

\section{Resultados}

\section{Obesidad y cáncer: evidencia epidemiológica}

Sólo del 5\% al $10 \%$ de todos los cánceres son causados por factores genéticos y hereditarios, mientras que del $90 \%$ al $95 \%$ se ha relacionado con factores de estilo de vida y medio ambiente. Casi el $30 \%$ de todos los cánceres han sido atribuidos al tabaquismo, 35\% a la dieta, $18 \%$ a las infecciones, $7 \%$ a la radiación y contaminantes del medio ambiente $(21,22)$.Estudios epidemiológicos sugieren que el exceso de peso corporal es responsable del $14 \%$ y el $20 \%$ de todas las muertes por cáncer tanto en hombre como en mujeres, respectivamente (22) (figura 1).

Figura 1. Porcentajes de las muertes por cáncer atribuidas a diferentes factores.

\section{causas de cáncer}

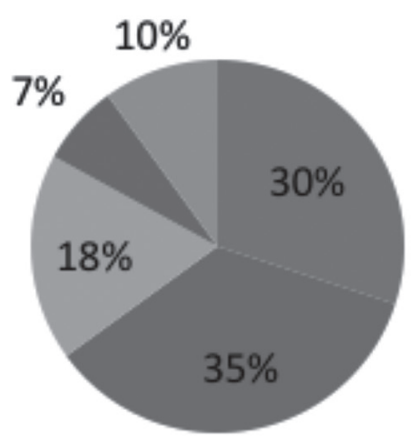

$$
\begin{aligned}
& 30 \% \text { - tabaquismo } \\
& 35 \% \text { - dieta } \\
& 18 \% \text { - infecciones } \\
& 7 \% \text { - radiación } \\
& 10 \% \text { - factores genéticos }
\end{aligned}
$$

Osler M, en el año 1987, determino que existía una asociación clara y reproducible entre la obesidad y el cáncer de mama y endometrio en mujeres posmenopáusicas, los estudios de cáncer de colon, recto, próstata y ovarios eran poco concluyentes (23).

En el año 2002, la Agencia Internacional para la Investigación del Cáncer (IARC) publicó el volumen 6 de los Manuales de Prevención del cáncer: el control del peso y la actividad física. El grupo de trabajo examinó estudios de casos y controles y estudios de cohorte de tipo prospectivos en cuanto a la asociación de cáncer con exceso de peso corporal, el estudio concluyó que hay pruebas suficientes de una asociación entre la obesidad y varios tipos de cáncer, tales como el cáncer de colon, mama en mujeres posmenopáusicas, endometrio, riñón y cáncer de esófago (24).

En el año 2003, Calle y colaboradores, publicaron un estudio que examinó una cohorte de 900.000 pacientes adultos en los Estados Unidos entre los años 1.982 y 1.998 , y determinaron que pacientes con obesidad mórbida tenían tasas más altas de mortalidad por cáncer que la población con un índice de masa corporal normal, aproximadamente un $52 \%$ y un $62 \%$ tanto en hombres como en mujeres, respectivamente (25).

En el año 2007, The International Agency for Research into Cancer and the World Cancer Research Fund (WCRF) concluyo, que los alimentos, la nutrición, la actividad física y los hábitos de vida saludable, son importantes en la modificación del proceso de cáncer. Los componentes de la dieta pueden proteger contra el cáncer, no solo antes de que comience el proceso, sino también durante y después de su desarrollo. Este reporte además concluyó que el aumento de la grasa corporal se asocia con un mayor riesgo en la presentación de cáncer de esófago, colon, recto, mama en mujeres posmenopáusicas y cáncer de endometrio, además menciona un riesgo importante para el cáncer de páncreas y una asociación probable entre el aumento de grasa corporal y el cáncer de vesícula biliar (26).

Renehan y colaboradores en el año 2008, determinaron que un aumento de $5 \mathrm{~kg} / \mathrm{m}^{2}$ en el IMC se asociaba fuertemente con la aparición de adenocarcinoma de esófago con un riesgo relativo (RR) de: 1.52, I.C 95\%,1.33 a 1.74, p < 0.0001, tiroides RR: 1.33, I.C 95\%, 1.04 a 1.70, p < 0.02, colon RR: 1.34, I.C 95\%, 1.20 a 1.28, p < 0.0001 y cáncer renal RR: 1.24, I.C 95\%, $1.15-1.34, \mathrm{p}<0.0001$. En las mujeres, se registraron fuertes asociaciones entre un aumento de $5 \mathrm{~kg} / \mathrm{m}^{2}$ en el IMC y el cáncer de endometrio RR: 1.59, I.C 95\%, 1.50 a 1.68, $\mathrm{p}<0.0001$, vesícula biliar RR: 1.59, I.C 95\%, 1.02 a 2.47, p $<0.04$, adenocarcinoma de esófago RR: 1.51, I.C 95\%, 1.31 a 1.74, p < 0.0001, y cáncer renal RR: 1,34,I.C 95\%, 1.25 a 1.43, p < 0.0001. También se demostró una asociación débil entre la obesidad y cáncer de mama en premenopáusicas, páncreas, tiroides, y cáncer de colon en las mujeres y el cáncer de recto y melanoma maligno en los hombres $(27,28)$, adicionalmente, Bhaskaran y cols (29), publicaron resultados muy similares en el Reino Unido el año 2014 a los encontrados por Renehan, donde reportaron una asociación positiva entre IMC elevados y el cáncer cervical RR: 1.10, I.C 99\%, 1.03 a 1.17; p = 0.00035. (Cuadro ) (29). 
Cuadro 1 . Tipos de cáncer asociados a obesidad.

\begin{tabular}{ccc}
\hline TIPOS DE CANCER & $\begin{array}{c}\text { RR CON IMC DE } \\
25-30 \mathrm{KG} / \mathrm{M} 2\end{array}$ & $\begin{array}{c}\text { RR CON IMC } \\
\text { DE } \geq 30 \text { KG/M2 }\end{array}$ \\
\hline endometrial & 2.0 & 3.5 \\
colorectal en hombres & 1.5 & 2.0 \\
colorectal en mujeres & 1.2 & 1.5 \\
mama (postmenopáusica) & 1.3 & 1.5 \\
esófago & 2.0 & 3.0 \\
(adenocarcinoma) & 1.5 & 2.5 \\
renal & 1.3 & 1.7 \\
pancreas & NO & $1.5-4.0$ \\
hígado & DETERMINADO & 2.0 \\
vesicular biliar & 1.5 & 2.0 \\
estomago & 1.5 & \\
(adenocarcinoma) & & \\
\hline
\end{tabular}

\section{Cáncer de colon y recto}

El aumento delos niveles de IMC y la circunferencia de cintura fueron asociados positivamente con el riesgo de cáncer de colon (30). Campbell y colaboradores determinaron que un incremento de $5 \mathrm{~kg} /$ $\mathrm{m} 2$, se asociaba positivamente con el riesgo de padecer cáncer de colon tanto en mujeres con un odds ratio (OR) de (1.20, I.C 95\%, 1.10 a 1.32) como para hombres OR: 1.30, I.C 95\%, 1.15a 1.47 (31). Qiwen Ben y colaboradores, en su estudio publicado en el año 2012, reportaron resultados muy similares a los encontrados por campbell. En general, un incremento de $5 \mathrm{~kg} / \mathrm{m} 2$ en el IMC, aumentaba en un 20 \% el riesgo de cáncer de colon es OR: 1.19, I.C 95\%, 1.13 a 1.26 (3234). La asociación fue ligeramente más fuerte para en el colon distal que en el colon proximal (54). Los estudios que han examinado la relación que hay entre el cáncer de recto y el IMC, indican que existe una asociación más reducida que la encontrada para el cáncer de colon $(24,27,35-37)$.

\section{Cáncer de mama}

El cáncer de mama es el segundo cáncer más común en el mundo y la neoplasia más común entre las mujeres. Lahmann y colaboradores en el 2004 determinaron que el riesgo relativo (RR) de desarrollar cáncer de mama en mujeres posmenopáusicas con un IMC de $28 \mathrm{~kg} /$ $\mathrm{m}^{2}$ o superior era de RR: 1.26, I.C 95\%, 1.09 a $1.46(38,39)$, además, se encontró que mujeres posmenopáusicas que no usaron terapia de reemplazo hormonal presentaban una asociación más fuerte de padecer cáncer de mama en comparación con aquellas mujeres que si recibieron el remplazo hormonal $(38,40)$. Cheraghi y cols en el 2.012, no encontraron una correlación significativa entre el IMC y el riesgo de cáncer de mama durante la premenopausia OR: 0.93, I.C 95\%, 0.86a $1.02(41,42)$.

\section{Cáncer de endometrio}

Friedenreich y colaboradores, en el año 2007 determinaron que, el riesgo relativo de desarrollar cáncer endometrial en mujeres obesas (IMC $\geq 30 \mathrm{~kg} / \mathrm{m} 2$ ) fue de RR: 1.78 , I.C $95 \%, 1.41$ a 2.26 , y para las mujeres con obesidad mórbida (IMC mayor o igual a 40) fue de RR: 3.02, I.C 95\%, 1.66 a 5.52, c (43 - 46). Cerca del 40\% de los cánceres endometriales son provocados por el exceso de peso.

\section{Cáncer de riñón}

El carcinoma de células renales es el tipo principal (80-90\%) de cáncer de riñón. El estudio IARC determinó que el IMC es un factor de riesgo para el desarrollo de cáncer de células renales tanto en hombres como en mujeres (24), además se ha observado que existe un mayor riesgo de padecer cáncer de células renales en el sexo femenino (RR: 1.06, IC $95 \%, 1.04$ a 1.07) (47-50).

\section{Cáncer de esófago}

El cáncer de esófago se subdivide en dos principales tipos histológicos: el carcinoma de células escamosas y el adenocarcinoma. El carcinoma de células escamosas del esófago es el tipo predominante de cáncer y ocurre principalmente en la parte superior y media del esófago, mientras que el adenocarcinoma del esófago ocurre con mayor frecuencia en la parte inferior.

El estudio IARC (24) determinó, que la obesidad y el sobrepeso aumentan el riesgo de padecer cáncer de esófago. El reflujo gastroesofágico se ha identificado como el principal factor de riesgo para el adenocarcinoma de esófago. El riesgo relativo para el adenocarcinoma de esófago asociado a sobrepeso y la obesidad fue de RR: 1.8, I.C 95\%, 1.48 a 2.19 y de RR: 2.58, I.C 95\%, 1.81 a 3.68 respectivamente, no se encontró asociación entre carcinoma de células escamosas y el IMC (51-53).

\section{Mecanismos fisiopatológicos}

La alteración en el peso corporal y la inactividad física pueden tener efectos notables sobre varios mecanismos fisiológicos que pueden posteriormente aumentar el riesgo de cáncer. Estos incluyen especialmente los efectos sobre el medio hormonal y la función inmune $(24,54)$, como sucede en los casos mediados por los estrógenos, insulina, factores de crecimiento insulínicos, leptina, adiponectina y los mediadores de la inflamación (figura 2) $(55,56)$.

Figura 2. Mecanismos biológicos de la relación entre obesidad y cáncer.

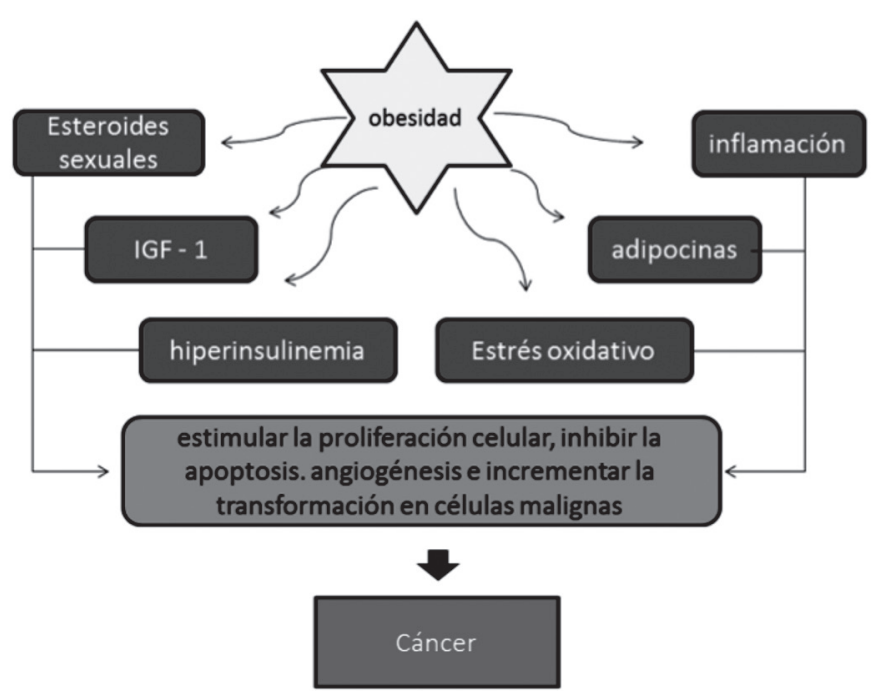

La obesidad, el sobrepeso, bajos niveles de actividad física y hábitos dietéticos occidentales favorecen niveles elevados de insulina circulantes, y se ha determinado que niveles elevados cumplen un papel importante en la tumorogenesis, ya que las células neoplásicas aprovechan este estado de hiperinsulinemia para estimular su proliferar, crecimiento y división celular (57). La hiperinsulinemia afecta a los factores de crecimiento insulinicos (IGF) elevando su efecto en el organismo; estos péptidos multifuncionales regulan la proliferación, diferenciación y la inhibición de la muerte celular (apoptosis), reduciendo además, las proteínas fijadoras de IGF 
(IGFBP-1) y (IGFBP-2) que están involucradas en la inhibición de las acciones del IGF, al prevenir su unión con el receptor de IGF-1(55, 58) y estimula la actividad tirosina kinasa de receptores de vías de crecimiento, como son el receptor de insulina (IR), el receptor de IGF-1 (IGF- 1R) y también el receptor híbrido de IGF-1/IR, los cuales activan la vía de la proteína kinasa activada por mitógenos (MAP) y la vía del fosfatidilinositide 3 kinasa/akt que se expresa en altas cantidades en células malignas.

El IGF-1 y la hiperinsulinemia inhiben la síntesis de globulina transportadora de hormonas sexuales (SHBG) que cumple la importante función de transportar la testosterona y el estradiol plasmático, lo que induce a que aumenten cantidades plasmáticas de esteroides sexuales libres. La resistencia a insulina y la hiperinsulinemia se han asociado con la patogénesis del cáncer colorectal (59), de mama en mujeres postmenopáusicas (60), próstata (61), y cáncer endometrial (62).

La exposición a los estrógenos durante las diferentes fases de la vida de una mujer puede influir en el aumento del riesgo de cáncer de endometrio (63) y mama (64). Después de la menopausia, cuando se suspende la producción de estrógeno ovárico, el tejido adiposo se convierte en la fuente principal de estrógeno endógeno a partir de precursores androgénicos. Mujeres postmenopáusicas obesas tienen mayores tasas de conversión de hormonas sexuales en comparación con mujeres postmenopáusicas no obesas $(65,66)$. En general, las mujeres con niveles circulantes de estrógeno superiores al 20\% de lo normal tienen entre 2 y 3 veces más riesgo de padecer cáncer (55, 65). Los esteroides sexuales son importantes mitógenos que pueden estimular la proliferación celular, inhibir la apoptosis e incrementar la transformación en células malignas (67).

El tejido adiposo es un órgano endocrino que produce y secreta una amplia gama de mensajeros químicos, tales como la adiponectina, factores angiogénicos, factores de crecimiento, citoquinas proinflamatorias (IL-1, TNF-alfa, IL-6) y la leptina, que participan en la mediación de enfermedades inflamatorias, la obesidad y desarrollo del cáncer (68).

La leptina, es la adipocina más estudiada y es un mediador que regula el apetito a nivel hipotalámico; la sobreexpresión crónica de la leptina induce la resistencia a la leptina, que resulta en el aumento de sus niveles circulante. El aumento de la Leptina tiene varios efectos protumorigénicos, en los cánceres de mama, de colon, endometrio y tienen efectos mitogenicos y antiapoptóticas en los cánceres de ovario y de próstata (69).

La adiponectina (APN) es una hormona secretada principalmente por el tejido adiposo y en un pequeño grado también es producida por los miocitos cardíacos, células de músculos y las células endoteliales. La adiponectina cumple funciones como anti-aterogénico, antiinflamatorio y tiene efectos sobre la sensibilidad a la insulina; el aumento de los niveles circulantes de adiponectina, o la activación de vías de señalización inducidas por la adiponectina, puede contrarrestar disfunciones metabólicas y hacer la progresión del cáncer más lento, su síntesis es reducida principalmente gracias a la acción de la leptina, la insulina y citocinas proinflamatorias asociado con la patogénesis del cáncer colorectal, endometrio, esófago, próstata y el cáncer de mama (70).

La obesidad genera un estado de inflamación y estrés oxidativo que tiende a la cronicidad (71), el papel de la inflamación en la tumorigénesis es muy aceptada, y se ha hecho evidente que un microambiente inflamatorio es un componente esencial en la aparición de los tumores, la inflamación se puede encontrar en todo el proceso de la tumorigénesis, desde el inicio hasta la promoción de tumores y finalmente hasta la metástasis $(69,71)$; la participación de citocinas locales como el FNT y citocinas sistémicas como la IL-6 están implicados en la angiogénesis, la supervivencia celular, el crecimiento, y la diferenciación de los tumores (72).

\section{Discusión}

La obesidad genera un elevado impacto en los sistemas de salud y en la calidad de vida de las personas tanto en países desarrollados como en vía de desarrollo (73). La prevalencia de esta epidemia varía ampliamente entre las diferentes regiones y países del mundo, debido a determinados factores que contribuyen en el almacenamiento de grasa corporal y el desequilibrio energético, tales como malos hábitos de vida, falta de actividad física, sedentarismo y el aumento de la oferta de calorías en los alimentos.

El 14 a $20 \%$ de las muertes por cáncer en adultos mayores de 50 años son atribuibles al sobrepeso y obesidad, y se estima que más de 90.000 muertes anuales por cáncer se evitarían si la población adulta mantuviera un IMC inferior a 25 durante toda su vida. Una tercera parte de todos los canceres son prevenibles, y otro tercio son curables si se diagnostican tempranamente, lamentablemente la falta de tecnología y políticas públicas enfocadas en el diagnóstico temprano de los estadios de cáncer, no lleva a que en el $75 \%$ de los países en desarrollo se diagnostique en estadios tumorales avanzados o incurables. Al realizar una comparación entre sujetos con peso normal y con sujetos obesos, los costos sanitarios aumentan considerablemente a medida que el IMC es mayor, gracias a sus altas tasas de mortalidad y la asociación con enfermedades crónicas no transmisibles y diversos tipos de cáncer (74).

Existen numerosos estudios que confirman que existe una asociación estadísticamente significativa entre el aumento del peso y los diferentes tipos de cáncer; además, se logra concluir, que a medida que aumenta el índice de masa corporal tanto en hombres como en mujeres también lo hace la asociación epidemiológica y la tasa de mortalidad. La obesidad y el sobrepeso pueden tener efectos notables sobre diferentes sistemas fisiológicos que pueden desencadenar el desarrollo neoplásico. No se ha encontrado ninguna asociación entre la obesidad y el cáncer de pulmón. En nuestro país no existe ningún estudio hasta la fecha que determine si existe alguna asociación entre la obesidad y los principales canceres que afectan a la población colombiana.

En Colombia el cáncer es la segunda causa de muerte después de las muertes asociadas a enfermedades en el sistema circulatorio. En el año 2010, el Legislativo colombiano declaró el cáncer como problema de salud pública de prioridad nacional con la promulgación de las Leyes 1384 y 1388 del año 2010. Así mismo, designa al Instituto Nacional de Cancerología como la instancia coordinadora del sistema de información de cáncer en todo el país con el fin de garantizar mejores medidas de vigilancia, prevención e intervención del cáncer en todos los grupos (75).

Es necesario que en Colombia se trabaje de manera intersectorial entre el gobierno, la academia, el sistema de salud y los profesionales de salud, la sociedad, la familia y la persona, para construir medidas más efectivas que permitan contribuir en la promoción de la salud y la prevención de la obesidad y el cáncer, garantizando así, la calidad de vida de la población colombiana. 


\section{Conclusión}

Existe una asociación entre la obesidad y la aparición de diversos tipos de cáncer, la comprensión de los mecanismos causales de esta asociación es importante para la implementación de medidas de salud pública y cambios en el estilo de vida, que busquen prevenir la aparición del cáncer y la obesidad.

La obesidad y el sobrepeso pueden tener efectos notables sobre varios sistemas fisiológicos que pueden posteriormente aumentar el riesgo de cáncer.

En nuestro país no existe ningún estudio hasta la fecha que determine si existe alguna asociación entre la obesidad y los principales canceres que afectan a la población colombiana.

\section{Conflictos de interés:}

Los autores declaramos no tener conflictos de interés en la publicación de este artículo.

\section{Referencias}

1. Rodríguez A, González B. El trasfondo económico de las intervenciones sanitarias en la prevención de la obesidad. Revista Española de Salud Pública. 2009;83(1):25-41.

2. Kelly T, Yang W, Chen CS, Reynolds K, He J. Global burden of obesity in 2005 and projections to 2030. International Journal of Obesity. 2008;32(9):1431-7.

3. Nguyen DM, El-Serag HB. The Epidemiology of Obesity. Gastroenterology Clinics of North America. 2010;39(1):1-7.

4. OMS | Obesidad y sobrepeso [Internet]. WHO. [Cited 2015 Feb 19]. Available from: http://www.who.int/mediacentre/ factsheets/fs311/es/index.html

5. Formiguera X, Cantón A. Obesity: epidemiology and clinical aspects. Best Practice \& Research Clinical Gastroenterology. 2004;18(6):1125-46.

6. Berghöfer A, Pischon T, Reinhold T, Apovian CM, Sharma AM, Willich SN. Obesity prevalence from a European perspective: a systematic review. BMC Public Health. 2008;8(1):200-2010.

7. Calle EE, Thun MJ. Obesity and cancer. Oncogene. 2004;23(38):6365-78.

8. Finucane MM, Stevens GA, Cowan MJ, Danaei G, Lin JK, Paciorek CJ, et al. National, regional, and global trends in bodymass index since 1980: systematic analysis of health examination surveys and epidemiological studies with 960 country-years and 9.1 million participants. The Lancet. 2011;377(9765):557-67.

1. Pischon $\mathrm{T}$, Nöthlings $U$, Boeing $H$. Obesity and cancer. Proceedings of the Nutrition Society. 2008;67(02):128-45.

2. Bray F, Jemal A, Grey N, Ferlay J, Forman D. Global cáncer transitions according to the Human Development Index (20082030): a population-based study. Lancet Oncol. 2012;13(8):790801.

3. Siegel R, DeSantis C, Jemal A. Colorectal cancer statistics, 2014: Colorectal Cancer Statistics, 2014. CA: A Cancer Journal for Clinicians. 2014;64(2):104-17.
4. Angarita F, Acuña S. Cáncer de seno: de la epidemiología al tratamiento. Univ. Méd.2008; 49(3):344-372.

5. Siegel R, Ma J, Zou Z, Jemal A. Cancer statistics, 2014: Cancer Statistics, 2014. CA: A Cancer Journal for Clinicians. 2014; 64(1):9-29

6. Ochoa FL, Montoya LP. Mortalidad por cáncer en Colombia 2005. Cancer mortality in Colombia in 2005. Revista de la facultad de medicina. 2009;57(4):304-315.

7. Milne FH, Judge DS, Preen DB, Weinstein P. Early life environment, life history and risk of endometrial cancer. Med Hypotheses. 2011;77(4):626-32.

8. García Ayala E, Sandoval Martínez DK, Calvo Castellanos JF, Lara Lozano JD. Adenocarcinoma oncocítico oxifílico de endometrio: reporte de caso. Revista Chilena de Obstetricia y Ginecología. 2013; 78(3):220-3.

9. Piñeros M, Gamboa O, Hernández-Suárez G, Pardo C, Bray F. Patterns and trends in cancer mortality in Colombia 1984-2008. Cancer Epidemiology. 2013;37(3):233-9.

10. Sanabria M, Umaña A, Serrano M, Sánchez M, Mesa J, Hernández G. Vías de carcinogénesis colorrectal y sus implicaciones clínicas. Rev colomb cancerol. 2012;16(3):170-181.

11. Angarita FA, TAWIL MM. Presentación inicial de las pacientes con diagnóstico de cáncer de seno en el Centro Javeriano de Oncología, Hospital Universitario San Ignacio. Revista Colombiana de Cirugía. 2010;25(1):19-26.

12. Perez J, Frisancho O. Cáncer de esófago: Características epidemiológicas, clínicas y patológicas en el hospital rebagliati. Rev. Gastroenterol. 2009;29(2):118-123.

13. Aggarwal BB, Vijayalekshmi RV, Sung B. Targeting inflammatory pathways for prevention and therapy of cancer: short-term friend, long-term foe. Clin Cancer Res 2009;15(2):425-430.

14. Anand P, Kunnumakara AB, Sundaram C, et al. Cancer is a preventable disease that requires major lifestyle changes. Pharmaceutical Research. 2008;25(9):2097-116.

15. Osler M. Obesity and cancer. A review of epidemiological studies on the relationship of obesity to cancer of the colon, rectum, prostate, breast, ovaries, and endometrium. Dan Med Bull. 1987;34(5):267-74.

16. Vainio H, Bianchini F (Eds.): IARC Handbooks of Cancer Prevent-Weight Control and Physical Activity. Lyon, France: IARC Press; 2002.

17. Calle EE, Rodríguez C, Walker- Thurmond K, Thun MJ. Overweight, obesity, and mortality from cancer in a prospectively studied cohort of U.S. adults. N Engl J Med. 2003;348(17):16251638 .

18. World Cancer Research Fund/American Institute for Cancer Research: Food, Nutrition, Physical Activity, and the Prevention of Cancer: a Global Perspective. Washington DC: AICR; 2007.

19. Renehan AG, Tyson M, Egger M, Heller RF, Zwahlen M. Bodymass index and incidence of cancer: a systematic review and meta-analysis of prospective observational studies. Lancet. 2008,371(9612):569-578.

20. Lichtman M. Obesity and the risk for a hematological malignancy: leukemia, lymphoma, or myeloma. Oncologist. 2010;15(10):1083-1101. 
21. Bhaskaran K, Douglas I, Forbes H, dos-Santos-Silva I, Leon DA, Smeeth L. Body-mass index and risk of 22 specific cancers: a population-based cohort study of 5.24 million UK adults. The Lancet. 2014; 384(9945):755-65.

22. Vucenik I, Stains JP. Obesity and cancer risk: evidence, mechanisms, and recommendations: Obesity and cancer. Annals of the New York Academy of Sciences. 2012;1271(1):37-43.

23. Patlak M, Nass S. The role of obesity in cancer survival and recurrence workshop summary. National Academies Press. 2012

24. Sánchez C, Ibáñez C, Klaassen J. Obesidad y cáncer: la tormenta perfecta. Rev Med Chile 2014; 142: 211-221.

25. Patterson RE, Rock CL, Kerr J, Natarajan L, Marshall SJ, Pakiz $\mathrm{B}$, et al. Metabolism and breast cancer risk: frontiers in research and practice. Journal of the Academy of Nutrition and Dietetics. 2013;113(2):288-96.

26. Arcidiacono B, Iiritano S, Nocera A, Possidente K, Nevolo MT, Ventura $\mathrm{V}$, et al. Insulin resistance and cancer risk: an overview of the pathogenetic mechanisms. Experimental Diabetes Research. 2012;2012:1-12.

27. Gunter MJ, Leitzmann MF. Obesity and colorectal cancer: epidemiology, mechanisms and candidate genes. The Journal of Nutritional Biochemistry. 2006 Mar;17(3):145-56.

28. Gaspard U. Hyperinsulinaemia, a key factor of the metabolic syndrome in postmenopausal women. Maturitas. 2009;62(4):362-5.

29. Aggarwal RR, Ryan CJ, Chan JM. Insulin-like growth factor pathway: A link between androgen deprivation therapy (ADT), insulin resistance, and disease progression in patients with prostate cancer? Urologic Oncology: Seminars and Original Investigations. 2013;31(5):522-30.

30. Papaioannou S, Tzafettas J. Anovulation with or without PCO, hyperandrogenaemia and hyperinsulinaemia as promoters of endometrial and breast cancer. Best Practice \& Research Clinical Obstetrics \& Gynaecology. 2010;24(1):19-27.

31. Persson I. Estrogens in the causation of breast, endometrial and ovarian cancers-evidence and hypotheses from epidemiological findings. The Journal of steroid biochemistry and molecular biology. 2000;74(5):357-64.

32. Chen WY. Exogenous and endogenous hormones and breast cancer. Best Practice \& Research Clinical Endocrinology \& Metabolism. 2008;22(4):573-85.

33. Siiteri PK. Adipose tissue as a source of hormones. Am J Clin Nutr. 1987; 45 (1): 277-282.

34. Van Kruijsdijk RCM, van der Wall E, Visseren FLJ. Obesity and cancer: The role of dysfunctional adipose tissue. Cancer Epidemiology, Biomarkers and Prevention. 2009;18(10):256978.

35. Kaaks R, Lukanova A, Kurzer MS. Obesity, endogenous hormones, and endometrial cancer risk: a synthetic review. Cancer Epidemiol Biomarkers Prev. 2002;11:1531-43

36. Jardé T, Perrier S, Vasson M-P, Caldefie-Chézet F. Molecular mechanisms of leptin and adiponectin in breast cancer. European Journal of Cancer. 2011;47(1):33-43.
37. Louie S, Roberts L, Nomura DK. Mechanism's linking obesity and cancer. Biochimica et biophysica acta. 2013;1831(10):1499508.

38. Hebbard L, Ranscht B. Multifaceted roles of Adiponectin in cancer. Best Practice and Research. Clinical Endocrinology and Metabolism. 2014;28(1):59-69.

39. Prieto-Hontoria PL, Pérez-Matute P, Fernández-Galilea M, Bustos M, Martínez JA, Moreno-Aliaga MJ. Role of obesityassociated dysfunctional adipose tissue in cancer: A molecular nutrition approach. Biochimica et Biophysica Acta (BBA) Bioenergetics. 2011;1807(6):664-78.

40. Cottam DR, Mattar SG, Barinas-Mitchell E, Eid G, Kuller L, Kelley DE, et al. The chronic inflammatory hypothesis for the morbidity associated with morbid obesity: implications and effects of weight loss. Obes Surg 2004;14(5):589-600.

41. Boeing H. Obesity and cancer - The update 2013. Best Practice and Research. Clinical Endocrinology and Metabolismm.2013;27(2):219-27.

42. Campbell PT, Jacobs ET, Ulrich CM, Figueiredo JC, Poynter JN, McLaughlin JR, et al. Case-control study of overweight, obesity, and colorectal cancer risk, overall and by tumor microsatellite instability status. Journal of the National Cancer Institute. 2010;102(6):391-400.

43. Ben Q, An W, Jiang Y, Zhan X, Du Y, Cai QC, et al. Body mass index increases risk for colorectal adenomas based on meta-analysis. Gastroenterology. 2012;142(4):762-72.

44. Moghaddam AA, Woodward M, Huxley R. Obesity and risk of colorectal cancer: a meta-analysis of 31 studies with 70,000 events. Cancer Epidemiology Biomarkers and Prevention. 2007;16(12):2533-47.

45. Ma Y, Yang Y, Wang F, Zhang P, Shi C, Zou Y, et al. Obesity and risk of colorectal cancer: a systematic review of prospective studies. Gorlova OY, editor. PLoS ONE. 2013;8(1):e53916.

46. Matsuo K, Mizoue T, Tanaka K, Tsuji I, Sugawara Y, Sasazuki S, et al. Association between body mass index and the colorectal cancer risk in Japan: pooled analysis of population-based cohort studies in Japan. Annals of Oncology. 2012. 23: 479-90.

47. Ma Y, Yang Y, Wang F, Zhang P, Shi C, Zou Y, et al. Obesity and risk of colorectal cancer: a systematic review of prospective studies. Gorlova OY, editor. PLoS ONE. 2013;8(1): e53916.

48. Reeves GK, Pirie K, Beral V, Green J, Spencer E, Bull D, et al. Cancer incidence and mortality in relation to body mass index in the Million Women Study: cohort study. BMJ. 2007;335(7630):1134-1134.

49. Lahmann PH, Hoffmann K, Allen $\mathrm{N}$ et al. Body size and breast cancer risk: Findings from the European prospective investigation into cancer and nutrition (EPIC). Int J Cancer. 2004:111(5),762-771.

50. DeSantis C, Ma J, Bryan L, Jemal A. Breast cancer statistics, 2013: Breast Cancer Statistics, 2013. CA: A Cancer Journal for Clinicians. 2014;64(1):52-62.

51. Carmichael A, Bates T. Obesity and breast cancer: a review of the literature. The Breast. 2004;13(2):85-92. 
52. Cheraghi Z, Poorolajal J, Hashem T, Esmailnasab N, Doosti Irani A. Effect of body mass index on breast cancer during premenopausal and postmenopausal periods: a meta-analysis. PLoS ONE. 2012;7(12), e51446

53. Makarem N, Chandran U, Bandera EV, Parekh N. Dietary fat in breast cancer survival. Annual Review of Nutrition. 2013; 33(1):319-48.

54. Arem H, Irwin ML. Obesity and endometrial cancer survival: a systematic review. International Journal of Obesity. 2013;37(5):634-9.

55. Friedenreich C, Cust A, Lahmann PH et al. Anthropometric factors and risk of endometrial cancer: the European prospective investigation into cancer and nutrition. Cancer Causes Control. 2007;18(4),399-413.

56. Chia V, Newcomb P, Trentham-Dietz A, Hampton J. Obesity, diabetes, and other factors in relation to survival after endometrial cancer diagnosis. Int J Gynecol Cancer. 2007;17(2):441-446.

57. Makarem N, Chandran U, Bandera EV, Parekh N. Dietary fat in breast cancer survival. Annual Review of Nutrition. 2013;33(1):319-48.

58. Sejnaui JE, Casasfranco DR, Ossa JA, Serrano CAA, Gutiérrez AF. Correlación entre tamaño, grado nuclear y supervivencia en el cáncer renal de células claras. Urología Colombiana. 2011;20(1):13-20.

59. Mathew A, George P, Ildaphonse G. Obesity and kidney cancer risk in women: a meta-analysis. Asian Pacific Journal of Cancer Prevention. 2009;10 (3): 471-8.

60. Bergström A, Hsieh CC, Lindblad P, Lu CM, Cook NR, Wolk A. Obesity and renal cell cancer-a quantitative review. British Journal of Cancer. 2001;85(7):984- 90.

61. Behrens G, Leitzmann MF. The association between physical activity and renal cancer: systematic review and meta-analysis. British Journal of Cancer. 2013;108(4):798-811.

62. Enzinger PC, Mayer RJ. Esophageal cancer. New england journal of medicine. 2003;349(23):2241-52.

63. Engeland A, Tretli S, Bjorge T. Height and body mass index in relation to esophageal cancer; 23-year follow-up of two million Norwegian men and women. Cancer Causes Control, 2004;15(8):837-843.

64. Kubo A, Corley D. Body mass index and adenocarcinomas of the esophagus or gastric cardia: a systematic review and metaanalysis. Cancer Epidemiology, Biomarkers and Prevention. 2006;15(5):872-8.

65. Thompson D, Wolf AM. The medical-care cost burden of obesity. Obesity Reviews. 2001;2(3):189-97.

66. Dee A, Kearns K, Ciaran O, Sharp L, Staines A, Victoria O, et al. The direct and indirect costs of both overweight and obesity: a systematic review. BMC. 2014;7(1):242-251.

67. Arias N. Registros poblacionales de cáncer: avances en Colombia, Chile y Brasil. Rev. Fac. Nac. Salud Pública 2013; 31(1): 127-135. 